\title{
Multi-facet Universal Schema
}

\author{
Rohan Paul* Haw-Shiuan Chang* Andrew McCallum \\ CICS, University of Massachusetts Amherst \\ \{rohpaul, hschang, mccallum\}@cs.umass.edu
}

\begin{abstract}
Universal schema (USchema) assumes that two sentence patterns that share the same entity pairs are similar to each other. This assumption is widely adopted for solving various types of relation extraction (RE) tasks. Nevertheless, each sentence pattern could contain multiple facets, and not every facet is similar to all the facets of another sentence pattern cooccurring with the same entity pair. To address the violation of the USchema assumption, we propose multi-facet universal schema that uses a neural model to represent each sentence pattern as multiple facet embeddings and encourage one of these facet embeddings to be close to that of another sentence pattern if they cooccur with the same entity pair. In our experiments, we demonstrate that multi-facet embeddings significantly outperform their singlefacet embedding counterpart, compositional universal schema (CUSchema) (Verga et al., 2016), in distantly supervised relation extraction tasks. Moreover, we can also use multiple embeddings to detect the entailment relation between two sentence patterns when no manual label is available.
\end{abstract}

\section{Introduction}

Relation extraction (RE) is a crucial step in automatic knowledge base construction (AKBC). A major challenge of $\mathrm{RE}$ is that the frequency of relations in the real world is a long-tail distribution but collecting sufficient human annotations for every relation is infeasible (Han et al., 2020).

Distant supervision is proposed to alleviate the issue (Mintz et al., 2009). Distant supervision assumes that a sentence pattern expresses a relation if the sentence pattern co-occurs with an entity pair and the entity pair has the relation. For example, we assume the sentence pattern " $\$ A R G 1$, the partner of fellow $\$ A R G 2$ " is likely to express the spouse

\footnotetext{
* indicates equal contribution
}

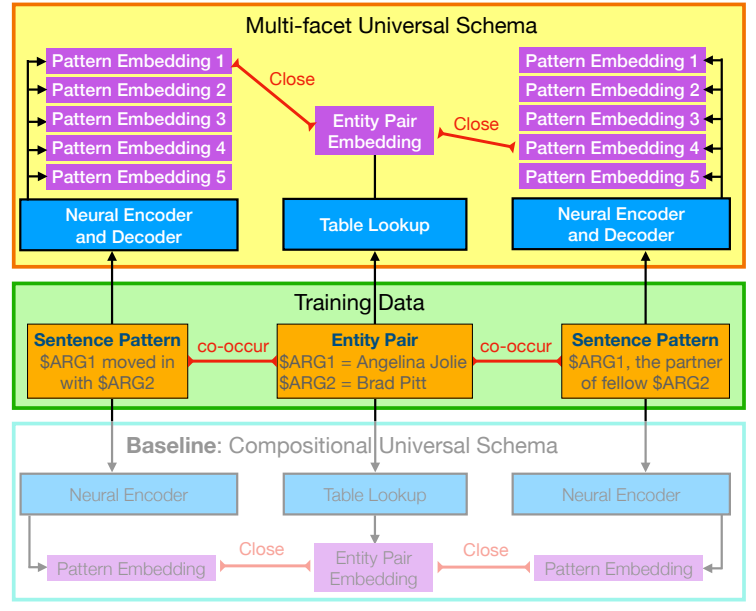

Figure 1: Comparison between the multi-facet and compositional universal schema. In our training loss, we encourage one of the facet embeddings from a sentence pattern to be similar to its co-occurred entity pair.

relation if we observe a text clip "... Angelina Jolie, the partner of fellow Brad Pitt ..." in our training corpus and a knowledge base tells us that Angelina Jolie and Brad Pitt has the spouse relation. Accordingly, we can infer that another entity pair is likely to have the spouse relation if we observe the text ", the partner of fellow" between them in a new corpus.

Universal schema (Riedel et al., 2013) extends this assumption by treating every sentence pattern as a relation, which means we assume that sentence patterns or relations in a knowledge base are similar if they co-occur with the same entity pair. For example, we assume " $\$ A R G 1$, the partner of fellow \$ARG2" and "\$ARG1, the wife of fellow $\$ A R G 2$ " are similar if they both co-occur with (Kristen Bell, Dax Shepard). Consequently, we can infer that " $\$ A R G 1$, the wife of fellow $\$ A R G 2$ " also implies spouse relation as " $\$ A R G 1$, the partner of fellow $\$ A R G 2$ " even if the knowledge base does not record the spouse relation between Kristen 


\section{Bell and Dax Shepard.}

Compositional universal schema (Verga et al., 2016) realizes the idea by using a LSTM (Hochreiter and Schmidhuber, 1997) to encode each sentence pattern into an embedding and encouraging the embedding to be similar to the embedding of the co-occurred entity pair. As in the lower part of Figure 1, the model makes the embeddings of two sentence patterns similar if they co-occur with the same entity pair. Baldini Soares et al. (2019) rely on a similar assumption and achieve state-of-theart results on supervised RE tasks by replacing the LSTM with a large pre-trained language model.

The variants of universal schema have many different applications, including multilingual RE (Verga et al., 2016), knowledge base construction (Toutanova et al., 2015; Verga et al., 2017), question answering (Das et al., 2017), documentlevel RE (Verga et al., 2018), N-ary RE (Akimoto et al., 2019), open information extraction (Zhang et al., 2019), and unsupervised relation discovery (Percha and Altman, 2018).

Nevertheless, one sentence pattern could contain multiple facets, and each facet could imply a different relation. In Figure 1, " $\$ A R G 1$, the partner of fellow $\$ A R G 2$ " could imply the entity pair has the spouse relation, the co-worker relation, or both. "\$ARG1 moved in with \$ARG2" could imply the spouse relation, the parent relation, ..., etc. If we squeeze the facets of a sentence pattern into a single embedding, the embedding is more likely to be affected by the irrelevant facets from other patterns co-occurred with the same entity pair (e.g., " $\$ A R G 1$ moved in with $\$ A R G 2$ " might incorrectly imply the co-worker relation).

Another limitation is that single embedding representation can only provide symmetric similarity measurement between two sentence patterns. Thus, an open research challenge is to predict the entailment direction of two sentence patterns only based on their co-occurring entity pair information.

To overcome the challenges, we propose multifacet universal schema, where we assume that two sentence patterns share a similar facet if they cooccur with the same entity pair. As in Figure 1, we use a neural encoder and decoder to predict multiple facet embeddings of each sentence pattern and encourage one of the facet embeddings to be similar to the entity pair embedding. As a result, the facets that are irrelevant to the relation between the entity pairs are less likely to affect the embeddings of entity pairs and other related sentence patterns. For example, the parent facet of "\$ARG1 moved in with $\$ A R G 2$ " could be excluded when updating the embeddings of (Angelina Jolie, Brad Pitt).

In our experiments, we first compare the multifacet embeddings with the single-facet embedding in distantly supervised RE tasks. The results demonstrate that multiple facet embeddings significantly improve the similarity measurement between the sentence patterns and knowledge base relations. Besides RE, we also apply multi-facet embeddings to unsupervised entailment detection tasks. In a newly collected dataset, we show that multi-facet universal schema significantly outperforms the other unsupervised baselines.

\section{Methods}

Our method is illustrated in Figure 2. In Section 2.1, we first provide our problem setup: We are given a knowledge base (KB) and a text corpus during training. Our goal is to extract relations by measuring the similarity between KB relations and an (unseen) sentence pattern or to detect entailment between two sentence patterns. In Section 2.2, we introduce our neural model, which predicts multifacet embeddings of each sentence pattern. Next, in Section 2.3, we describe our objective function, which encourages the embeddings of co-occurred entity pairs to be close to the embeddings of their closest pattern facets. Finally, in Section 2.4, we explain that multi-facet embeddings could be viewed as the cluster centers of possibly co-occurred entity pairs, and in Section 2.5, we provide our scoring functions for distantly supervised RE and unsupervised entailment tasks.

\subsection{Background and Problem Setup}

Our RE problem setup is the same as compositional universal schema (Verga et al., 2016). First, we run named entity recognition (NER) and entity linking on a raw corpus. After identifying the entity pairs in each sentence, we prepare a co-occurrence matrix as in Figure 2. Similarly, we represent the KB relations between entity pairs as a co-occurrence matrix and merge the matrices from the $\mathrm{KB}$ and the training corpus. The merged matrix has $y_{i, j}=1$ if the $i$ th sentence pair or KB relation co-occurs with the $j$ th entity pair and $y_{i, j}=0$ otherwise.

During testing, we use NER to extract an entity pair and the sentence pattern, which might not have been seen in the training corpus. Next, we extract 


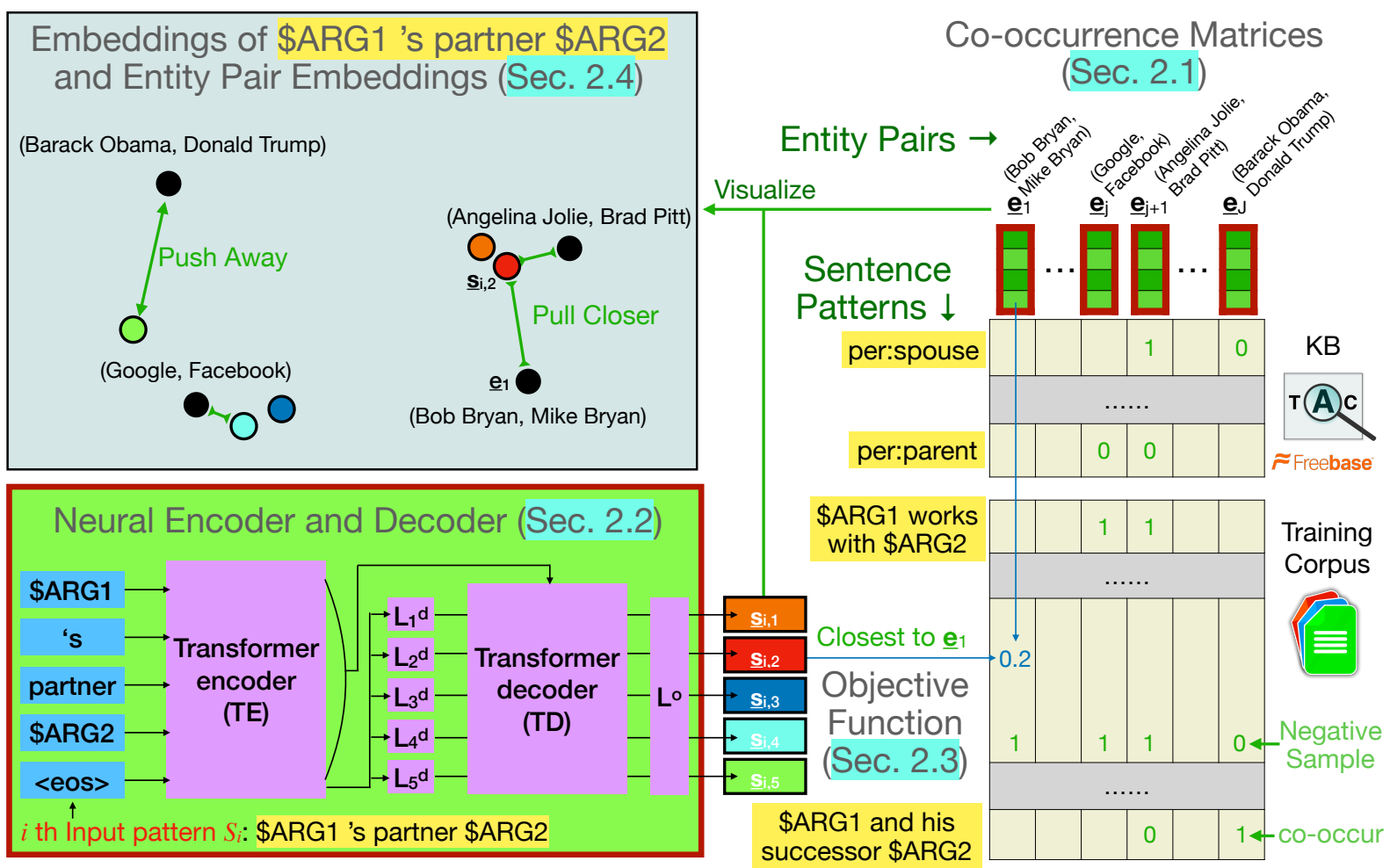

Figure 2: An illustration of the proposed method. The training signal comes from the co-occurrence matrices of the $\mathrm{KB}$ and training text corpus on the right. On the lower left, we visualize our neural encoder, which captures the compositional meaning of tokens in the sentence pattern, and our neural decoder, which models the dependency among multiple facet embeddings. When a sentence pattern co-occurs with an entity pair, the training loss minimizes the distance between the entity pair embedding and the closest facet embedding of the sentence pattern (e.g., 0.2 between $s_{i, 2}$ and $e_{1}$ ). Trainable parameters in our model are highlighted using red borders. On the upper left, we visualize the embedding space to establish the connection between our method and clustering.

relations by computing the similarity between the sentence pattern embeddings and the embeddings of the applicable KB relations. Besides RE, we also detect the entailment between two sentence patterns by comparing their embeddings.

\subsection{Neural Encoder and Decoder}

We use a neural model to predict $K$ facet embeddings of each sentence pattern. The goal is similar to Chang et al. (2021), which predict a fixed number of embeddings of a sentence, so we adopt their neural model as shown in Figure 2.

For the $i$ th sentence pattern $S_{i}$, we append an $\langle$ eos $>$ to its end and use a 3-layer Transformer (Vaswani et al., 2017) encoder $T E$ to model the compositional meaning of the input word sequence: $\underline{\boldsymbol{u}}_{i, 1} \ldots \underline{\boldsymbol{u}}_{i,\left|S_{i}\right|} \underline{\boldsymbol{u}}_{i,<\operatorname{eos}>}=T E\left(S_{i}<\operatorname{eos}>\right)$, where $\underline{\boldsymbol{u}}_{i, l}$ is an embedding contextualized by the encoder. In the experiment, we also replace the Transformer with a bidirectional LSTM (bi-LSTM) to show that the improvement of multi-facet embeddings is independent of the encoder choice.
The embedding $\underline{\boldsymbol{u}}_{i,<\text { eos }>}$ represents the whole sentence pattern; we use $K$ different linear layers $L_{k}^{d}$ to transform the embedding into the inputs of our decoder: $\underline{\boldsymbol{b}}_{i, k}=L_{k}^{d}\left(\underline{\boldsymbol{u}}_{i,<\operatorname{eos}>}\right)$.

The facets in a sentence pattern often have some dependency. For example, the patterns that express the partnership between two people might also express the collaboration relation between two companies. To leverage the dependency, we use another 3-layer Transformer as our decoder $T D$. Besides the self-attention, we allow the hidden states in the decoder to query the contextualized word embeddings $\underline{\boldsymbol{u}}_{i, l}$ from the encoder (Vaswani et al., 2017) and output the embeddings corresponding to the different facets $\underline{\boldsymbol{d}}_{i, k}: \underline{\boldsymbol{d}}_{i, 1} \ldots \underline{\boldsymbol{d}}_{i, K}=$ $T D\left(\underline{\boldsymbol{b}}_{i, 1} \ldots, \underline{\boldsymbol{b}}_{i, K}, \underline{\boldsymbol{u}}_{i, 1} \ldots \underline{\boldsymbol{u}}_{i,<\mathrm{eos}>}\right)$. Notice that we do not use autoregressive decoding as in Vaswani et al. (2017), so our decoder could also be viewed as another encoder with attention to the output of the encoder $T E$. Finally, to convert the hidden state size to the entity embedding size, we let the outputs of decoder go through another linear layer 
$L^{o}$ to get the facet embedding (i.e., sentence pattern embedding): $\underline{\boldsymbol{s}}_{i, k}=L^{o}\left(\underline{\boldsymbol{d}}_{i, k}\right)$.

\subsection{Objective Function}

When measuring the distance between the $j$ th entity pair and the $i$ th sentence pattern, we compute the Euclidean distance between the entity pair embedding $\underline{\tilde{e}}_{j}$ and its closest facet embedding of the $i$ th sentence pattern. The distance is defined as

$$
D\left(\left\{\underline{\boldsymbol{s}}_{i, k}\right\}_{k=1}^{K}, \underline{\tilde{e}}_{j}\right)=\min _{k=1}^{K} \min _{0 \leq \eta_{k} \leq 1}\left\|\underline{\tilde{e}}_{j}-\eta_{k} \underline{\boldsymbol{s}}_{i, k}\right\|^{2},
$$

where the entity pair embedding is normalized (i.e., $\left\|\underline{\tilde{e}}_{j}\right\|=1$ ). During testing, we ignore the magnitude of facet embeddings, so we use $\eta_{k}$ to eliminate the magnitude of facet embeddings $\underline{s}_{i, k}$ during training. We do not allow negative $\eta_{k}$ to prevent the gradient flow from pushing $\underline{s}_{i, k}$ toward the inverse direction of $\underline{\tilde{e}}_{j}$ and we ensure $\eta_{k} \leq 1$ to avoid the neural model from outputting $\underline{s}_{i, k}$ with a very small magnitude.

As in Figure 2, we minimize the distance $D\left(\left\{\underline{\boldsymbol{s}}_{i, k}\right\}_{k=1}^{K}, \underline{\tilde{e}}_{j}\right)$ in our loss function when the $i$ th sentence pair co-occurs with the $j$ th entity pair (i.e., $y_{i, j}=1$ ). For negative samples (i.e., $y_{i, j}=0$ ), we maximize the distance instead. That is, the major term of our loss function is defined as

$$
\sum_{(i, j) \in R}\left(2 \cdot y_{i, j}-1\right) r_{i, j} D\left(\left\{\underline{\boldsymbol{s}}_{i, k}\right\}_{k=1}^{K}, \underline{\tilde{e}}_{j}\right),
$$

and the other regularization term $\Omega$ in the loss function will be described in the appendix. $R$ is a set that includes all positive and negative samples. Positive samples are $(i, j)$ such that $y_{i, j}=1$ and the negative samples are constructed by pairing a randomly selected sentence pattern with the $j$ th entity pair. To balance the influence of popular entity pairs (i.e., entity pairs that co-occur with many sentence patterns) and rare entity pairs on our model, we set the weight of each pair, $r_{i, j} \propto \frac{1}{\sum_{i} y_{i, j}}$ and $\frac{\sum_{(i, j) \in R} r_{i, j}}{|R|}=1$.

We generate the embeddings for KB relations in a similar way. We use a single token to represent the relation and append an $<$ eos $>$ (e.g., per:spouse $<\operatorname{eos}>$ ) to form the input of our neural model. The $\mathrm{KB}$ relations usually co-occur with more entity pairs, so we set the number of facet embeddings for KB relations $K_{r e l}$ to be larger than the number of facet embeddings for sentence patterns $K$.

\subsection{Connection to Clustering}

If a sentence pattern contains multiple facets that describe different relations between the entity pairs, the pattern often co-occurs with different kinds of entity pairs. For example, " $\$ A R G 1$ 's partner $\$ A R G 2$ " in Figure 2 could express the collaboration relationship between two companies or the partnership between two people, so the sentence patterns could co-occur with two companies such as (Google, Facebook) and two people such as (Bob Bryan, Mike Bryan).

Different kinds of entity pairs often have very different embeddings, so we could discover the facets of sentence patterns by clustering the embeddings of entity pairs. Here, a facet refers to a mode of the embedding distribution of the entity pairs that could possibly co-occur with the sentence pattern. A facet could be represented by multiple facet embeddings and each facet embedding corresponds to a cluster center of the entity pair embeddings. Hence, although the number of facet embeddings $K$ is fixed for all the sentence patterns, our model can capture the facets of the sentence patterns well when the number of facets is less than $K$.

In equation 1, we choose the closest facet embedding of the sentence pattern for each co-occurring entity pair embedding and minimize their distance. For example, $\underline{\boldsymbol{s}}_{i, 2}$ and the embedding of (Bob Bryan, Mike Bryan) are pulled closer in Figure 2. Minimizing equation 1 by passing the gradient through the scaled facet embedding $\eta_{k} \underline{s}_{i, k}$ is the same as minimizing a Kmeans loss, so the loss term induced by positive sample pairs encourage each $\underline{s}_{i, k}$ to become the cluster center of its nearby co-occurring entity pair embeddings. The details of our training algorithm could be found in the appendix.

The co-occurrence matrices in RE tasks are usually extremely sparse, and most of the sentence patterns only co-occur with a few entity pairs, which makes it difficult to derive multiple high-quality embeddings by clustering the co-occurring entity pair embeddings as in multi-sense word embedding methods such as Neelakantan et al. (2014). The proposed method solves this sparsity challenge by predicting the cluster centers using a neural model. For instance, even if " $\$ A R G 1$ 's partner $\$ A R G 2$ " does not co-occur with many entity pairs, its embeddings are encouraged to be close to the embeddings of entity pairs that co-occur with other similar patterns (e.g., “\$ARG1 and her partner $\$ A R G 2$ "). 

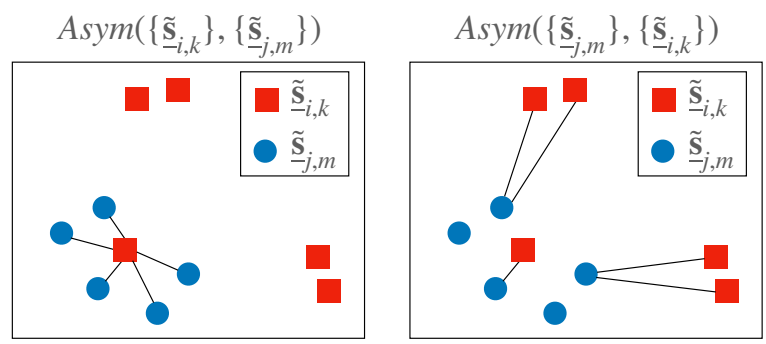

Figure 3: Comparison of the asymmetric similarities. $\operatorname{Asym}\left(\left\{\underline{\tilde{s}}_{i, k}\right\},\left\{\underline{\tilde{s}}_{j, m}\right\}\right)>\operatorname{Asym}\left(\left\{\underline{\tilde{s}}_{j, m}\right\},\left\{\underline{\tilde{s}}_{i, k}\right\}\right)$ because the average cosine distance on the left is smaller than that on the right.

\subsection{Scoring Functions}

In compositional universal schema, the similarity between the $i$ th and $j$ th sentence patterns are measured by the symmetric cosine similarity $\underline{\tilde{s}}_{i, 1}^{T} \underline{\tilde{s}}_{j, 1}$, where $\underline{\tilde{s}}_{i, 1}=\frac{\underline{s}_{i, 1}}{\left\|\underline{s}_{i, 1}\right\|}$. When using multiple embeddings to represent a sentence pattern, we can compute the asymmetric similarity as

$$
\operatorname{Asym}\left(\left\{\tilde{\underline{s}}_{i, k}\right\},\left\{\underline{\tilde{s}}_{j, m}\right\}\right)=\frac{\sum_{m=1}^{K} \max _{k=1}^{K}\left(\tilde{\underline{s}}_{i, k}^{T} \tilde{\underline{s}}_{j, m}\right)}{K} .
$$

In an example of Figure 3, a red square $\underline{\underline{s}}_{i, k}$ is close to all the blue points, which leads to a high $\operatorname{Asym}\left(\left\{\underline{\tilde{s}}_{i, k}\right\},\left\{\underline{\tilde{s}}_{j, m}\right\}\right)$.

Between two sentence patterns with entailment relation, we empirically find that the embeddings of a premise (the more specific pattern) often have some facet embeddings that are far away from all the embeddings of its hypothesis (the more general pattern). Relying on the tendency, we could detect the direction of the entailment relation. For example, the $i$ th sentence pattern (red squares) in Figure 3 is more likely to be premise if the $i$ th and $j$ th (blue circles) sentence patterns have an entailment relation.

We suspect the reason is that more specific patterns could contain more words that are similar to the words of other patterns expressing different relations. For example, " $\$ A R G 1$, the wife of fellow $\$ A R G 2$ " have a facet embedding for spouse relation and another facet embedding for the co-worker relation because the pattern has high word overlapping with " $\$ A R G 1$, the wife of $\$ A R G 2$ " and "\$ARG1 and her fellow \$ARG2". Another possible reason is that the articles in our corpus tend to use more specific patterns to express the relation between a pair of entities (Shwartz et al., 2017).

When performing RE, we compute the symmetric similarity between $i$ th sentence pattern and $j$ th
KB relation $\operatorname{Sim}\left(\left\{\underline{\tilde{s}}_{i, k}\right\},\left\{\underline{\tilde{s}}_{j, m}\right\}\right)$ by

$$
\frac{\operatorname{Asym}\left(\left\{\tilde{\tilde{s}}_{i, k}\right\},\left\{\underline{\tilde{s}}_{j, m}\right\}\right)+\operatorname{Asym}\left(\left\{\underline{\tilde{s}}_{j, m}\right\},\left\{\underline{\tilde{s}}_{i, k}\right\}\right)}{2} .
$$

\section{Experiments}

We primarily compare our method with compositional universal schema (CUSchema) (Verga et al., 2016) because CUSchema is one of the state-of-theart RE methods in the small model regime (without using large pre-trained language models) (Chang et al., 2016; Chaganty et al., 2017). ${ }^{1}$

In Section 3.1, we visualize and analyze the facet embeddings. Next, we use distant-supervised RE tasks to evaluate our symmetric similarity measurement in Section 3.2, and detect entailment between sentence patterns to evaluate our asymmetric similarity measurement in Section 3.3.

\subsection{Embedding Visualization}

We visualize the embeddings of sentence patterns and a KB relation from the single embedding model and multi-facet embedding model that perform the best in the RE tasks (i.e., Ours (Single-Trans) and Ours (Trans) in Table 1). We project the facet embeddings to a 2-dimensional space using multidimensional scaling (MDS) (Borg and Groenen, 2005) and visualize the embeddings of one KB relation and three related sentence patterns in Figure 4. The three sentence patterns are selected from our validation set, so the model is not aware of the entity pairs that actually co-occur with the patterns during training. For each facet embedding, we show two among five of its closest entity pairs to visualize the meaning of the embedding space. ${ }^{2}$

\footnotetext{
${ }^{1}$ We have not yet applied the multi-facet embeddings approach to the models that rely on a large pretrained language model (LM) (Baldini Soares et al., 2019) due to computational and evaluation considerations. Computationally speaking, training state-of-the-art models requires intensive GPU resources. Besides, a smaller model size might be desired when we need to construct a knowledge base from a large corpus in real time. Moreover, there is no existing pretrained LM in some domains (Zhang et al., 2019), and training the $\mathrm{LM}$ in a new domain from scratch requires even more GPU resources.

In terms of the evaluation consideration, our method is an improvement over CUSchema, so we want to compare it with CUSchema fairly. Furthermore, evaluating entailment between two full sentences is more difficult than between the sentence patterns, and we are not aware of a LM-based model that only considers the text between the entity pairs.

${ }^{2}$ Notice that our training signal is sparse and noisy and the projection does not necessarily preserve the original distances, so the entity pairs with similar relations might be relatively far away from each other.
} 

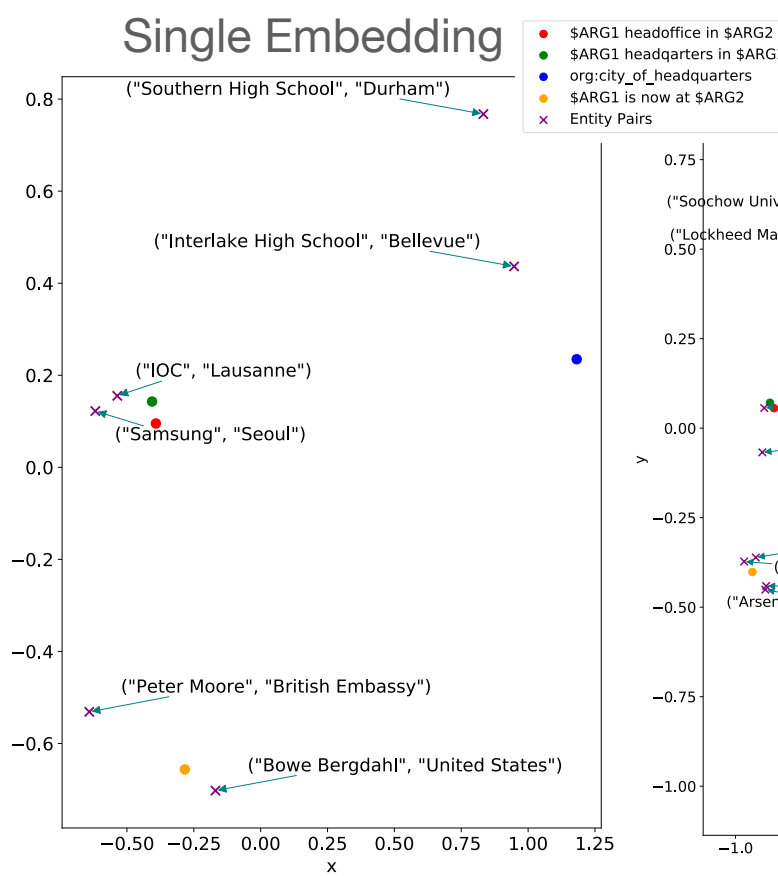
\$ARG1 headqarters in \$ARG2 org:city_of_headquarters
SARG1 is now at \$ARG2 Entity Pairs

Figure 4: Facet embedding visualization of Ours (Single-Trans) on the left and Ours (Trans) on the right. Dots are the facet embeddings outputted by our models and crosses are their nearby entity pair embeddings

In the single embedding model, the embedding of org:city_of_headquarter is close to the embedding of (school, location) while " $\$ A R G 1$ headoffice in $\$ A R G 2$ " is close to (company, location) and “\$ARG1 headquarter in \$ARG2”.

In the multi-facet embedding model, some embeddings of org:city_of_headquarter are closer to (school, location) and others are closer to (company, location). In addition to these entity pairs, " $\$ A R G 1$ headoffice in \$ARG2" and "\$ARG1 headquarter in $\$ A R G 2$ " also co-occur with (people, location) and (people/organization, year). Using the visualization of multi-facet embedding, we can understand which facets of org:city_of headquarter are similar or dissimilar to "\$ARG1 headoffice in \$ARG2", which cannot be done if all facets are averaged into a single embedding as in the traditional models.

The facet embeddings of " $\$ A R G 1$ is now at $\$ A R G 2$ " are close to (people, organization) where the organization could be school, sports team, and company. Using multiple embeddings could avoid enforcing the closeness of these entity pairs with different relations. The results also indicate that our model can output reasonable cluster centers despite learning from the sparse and noisy training data. Finally, we can see that if a sentence pattern has fewer facets than $K$, our model learns to output some very similar facet embeddings, which makes the performance less sensitive to the setting of $K$.
Multi-facet Embedding

("McDonald's", "Oak Brook")

"Interlake High'schól's "Bellevue")

m Organization", "Madrid")

("Eastern Kentucky University", "Richmond")

("International Olympic Committee", "2007")

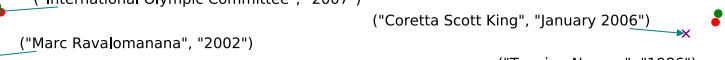

("Tenzing Norgay", "1986")

("Martin Schempp", "Stuttgart")

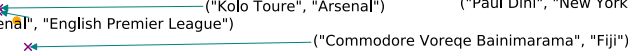

« ("Stephen Roach", "Morgan Stanley")

("Robert F. Goheen", "Princeton University")

("Arabinda Rajkhowa", "United Liberation Front of Assam")

* ("Derek Abbott", "University of Adelaide")

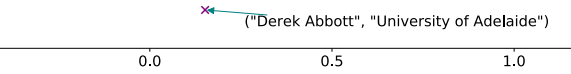

\subsection{Relation Extraction}

We follow the same training data and testing protocol in compositional universal schema (CUSchema) (Verga et al., 2016) ${ }^{3}$ to highlight the benefit of predicting multiple facet embeddings, and the relation extraction step in TAC KBP slot-filling tasks is used to compare the different models.

Setup: The training data for our RE models are prepared by distant supervision without requiring any manually labeled data. The relations in Freebase (Bollacker et al., 2008) are mapped to TAC relations (e.g., org:city_of_headquarter) and the NER tagger and entity linker are run in a raw text corpus. Then, the training data is cleaned using the methods in Roth et al. (2013).

During testing, we are given a query containing the head entity and a query TAC relation in the slot-filling tasks, and the goal is to extract the tail entity from the candidate sentences. The NER tagger and query expansion are used to gather the candidate sentence patterns, and we compute the similarity scores from different models between the candidate sentence patterns and query relation. Finally, we compare the extracted second entity with the ground truth using exact string matching and report the precision, recall, and F1 scores.

Following Verga et al. (2016), we use TAC 2012

\footnotetext{
${ }^{3}$ https://github. com/patverga/ torch-relation-extraction
} 


\begin{tabular}{|c|ccc|ccc|ccc|}
\hline \multirow{2}{*}{ Method } & \multicolumn{3}{|c|}{ TAC 2012 (Validation) } & \multicolumn{3}{c|}{ TAC 2013 } & \multicolumn{3}{c|}{ TAC 2014 } \\
\cline { 2 - 9 } & Prec & Recall & F1 & Prec & Recall & F1 & Prec & Recall & F1 \\
\hline USchema* & $\mathbf{3 4 . 8}$ & 23.7 & 28.2 & $\mathbf{4 2 . 6}$ & 29.4 & 34.8 & $\mathbf{3 5 . 5}$ & 24.3 & 28.8 \\
CUSchema (LSTM)* & 27.0 & $\mathbf{3 2 . 7}$ & 29.6 & 39.6 & 32.2 & 35.5 & 32.9 & 27.3 & 29.8 \\
Ours (Single-LSTM) & 25.7 & 21.7 & 23.5 & 30.4 & 26.3 & 28.2 & 22.1 & 20.5 & 21.3 \\
Ours (Single-Trans) & 26.1 & 21.6 & 23.7 & 29.5 & 25.2 & 27.2 & 19.0 & 21.2 & 20.0 \\
Ours (LSTM) & 32.0 & 28.9 & 30.3 & 41.3 & $\mathbf{3 3 . 9}$ & 37.2 & 34.1 & $\mathbf{2 9 . 5}$ & $\mathbf{3 1 . 6}$ \\
Ours (Trans) & 33.6 & 27.7 & $\mathbf{3 0 . 4}$ & 42.5 & 33.2 & $\mathbf{3 7 . 3}$ & 34.6 & 28.5 & 31.3 \\
\hline USchema + CUSchema (LSTM)* & 29.3 & 32.8 & 30.9 & $\mathbf{4 1 . 9}$ & 34.4 & 37.7 & 29.3 & 34.1 & 31.5 \\
USchema + Ours (LSTM) & 29.2 & 33.7 & 31.3 & 38.1 & $\mathbf{3 8 . 9}$ & 38.5 & 31.5 & $\mathbf{3 4 . 4}$ & 32.9 \\
USchema + Ours (Trans) & $\mathbf{3 0 . 4}$ & $\mathbf{3 3 . 9}$ & $\mathbf{3 2 . 1}$ & 39.0 & 38.8 & $\mathbf{3 8 . 9}$ & $\mathbf{3 2 . 0}$ & 34.0 & $\mathbf{3 3 . 0}$ \\
\hline
\end{tabular}

Table 1: Distantly supervised relation extraction using different versions of the universal schema. All numbers are $\%$. CUSchema refers to compositional universal schema. Trans is an abbreviation of Transformer. The best scores of the single models and ensemble models are highlighted. *The performance of TAC 2013 and 2014 are copied from Verga et al. (2016).

as our validation set to determine the threshold score for each TAC relation. Each model's hyperparameters are tuned separately using the validation set (TAC 2012) to ensure a fair comparison.

We compare the following methods:

Ours (Trans): Our method that measures the similarity between the sentence pattern $\left\{\underline{\tilde{s}}_{i, k}\right\}$ and TAC relation $\left\{\underline{\tilde{s}}_{j, m}\right\}$ using $\operatorname{Sim}\left(\left\{\underline{\tilde{s}}_{i, k}\right\},\left\{\underline{\tilde{s}}_{j, m}\right\}\right)$ in equation 4. Trans is an abbreviation of the Transformer encoder. We set $K=5$ and $K_{\text {rel }}=11$ based on the validation set.

Ours (LSTM): The same as Ours (Trans) except that we use bi-LSTM as our encoder instead.

Ours (Single-*): Our methods that use single facet embedding to represent each sentence pattern or KB relation. When setting $K=K_{\text {rel }}=1$, our decoder becomes the interleaving feedforward layers and cross-attention layers attending to the output embeddings of the encoder.

CUSchema (LSTM): Compositional universal schema (Verga et al., 2016). The method is similar to Ours (Single-LSTM) but uses a different loss function, neural architecture (no decoder), and hyperparameter search procedure.

USchema: Universal schema (Riedel et al., 2013) estimates every sentence pattern embedding by factorizing the co-occurrence matrices (i.e., replacing the bi-LSTM in CUSchema with a look-up table). USchema + *: Verga et al. (2016) show that taking the maximal similarity between USchema and CUSchema model improves the F1. We also apply the same merging procedure to our model.

Results: In Table 1, the proposed method Ours (Trans) significantly outperform CUSchema (LSTM) before and after combining with universal schema. As far as we know, our proposed multi- facet embedding is the first method that outperforms compositional universal schema using the same training signal in the distant-supervised RE benchmark they proposed.

Although the recall of USchema is low because it does not exploit the similarity between the patterns (e.g., " $\$ A R G 1$ happily married $\$ A R G 2$ " is similar to "\$ARG1 married \$ARG2"), USchema has a high precision because it also won't be misled by the similarity (e.g., " $\$ A R G 1$, and his wife $\$ A R G 2$ " expresses the spouse relation but “\$ARG1, his wife, and \$ARG2" does not) (Verga et al., 2016). Thus, combining USchema and Ours (Trans) leads to the best performance.

Ours (Trans) and Ours (LSTM) perform similarly. Furthermore, Ours (LSTM) performs much better than Ours (Single-LSTM), which demonstrates the effectiveness of using multiple embeddings. Notice that multiple facet embeddings could improve the performance even after the training data have been cleaned. This indicates that our method is complementary to the noise removal methods in Roth et al. (2013).

\subsection{Entailment Detection}

Entailment is a common and fundamental relation between two sentence patterns. Some examples could be seen in Table 2. Unsupervised hypernym detection (i.e., entailment at the word level) is extensively studied (Shwartz et al., 2017), but we are not aware of any previous work on unsupervised entailment detection at the sentence level, nor any existing entailment dataset between sentence patterns. Thus, we create one.

Dataset Creation: We use WordNet (Miller, 1998) to discover the entailment candidates of sen- 


\begin{tabular}{|c|c|c|c|c|c|c|}
\hline Premise (Specific Pattern) & Hypothesis (General Pattern) & Label & Ours & CUSchema & Ours Diff & Freq Diff \\
\hline \$ARG1, the president of the \$ARG2 & \$ARG1, the leader of the \$ARG2 & Entailment & 0.98 & 0.94 & + & + \\
\hline \$ARG1 's chairman , \$ARG2 & \$ARG1 's leader, \$ARG2 & Entailment & 0.95 & 0.87 & + & - \\
\hline \$ARG1 's father, \$ARG2 & \$ARG1 's leader, \$ARG2 & Other & 0.08 & 0.52 & NA & NA \\
\hline \$ARG1 worked with \$ARG2 & \$ARG1 deal with \$ARG2 & Entailment & 0.92 & 0.83 & + & - \\
\hline \$ARG1 had with \$ARG2 & \$ARG1 deal with \$ARG2 & Other & 0.96 & 0.88 & NA & NA \\
\hline \$ARG1 said the \$ARG2 & \$ARG1 say the \$ARG2 & Paraphrase & 0.93 & 0.92 & NA & NA \\
\hline
\end{tabular}

Table 2: Example of sentence pattern pairs, its label, and our predictions in our entailment experiment. Ours and Ours Diff are the predictions from Ours (Trans). Freq Diff is the frequency difference baseline.

tence pattern pairs and manually label the candidates. For each sentence pattern in the training data of Verga et al. (2016), we replace one word at a time with its hypernym based on the WordNet hierarchy. The two sentence patterns before and after replacement form an entailment candidate.

We label 1,500 pairs of the most popular sentence pattern, which co-occurs with the highest number of unique entity pairs. Each candidate could be labeled as entailment, paraphrase, or other. Finally, around $20 \%$ of the candidates are randomly chosen to form the validation set, and the rest are in the test set. More details of the dataset creation process could be seen in the appendix

In this dataset, only $22 \%$ and $10 \%$ of candidates are labeled as entailment and paraphrase, respectively. This suggests that entailment relation between two sentence patterns is hard to be inferred by only the hypernym relation (i.e., entailment relation at the word level) in WordNet.

Setup: We evaluate entailment detection using the typical setup and metrics in hypernym detection (Shwartz et al., 2017). Negative examples include the candidates labeled as paraphrases and others. We compare the average precision of different methods (i.e., AUC in the precision-recall curve) (Hastie et al., 2009). In addition, we predict the direction of entailment relation in each pair (i.e., which pattern is the premise) and report the accuracy. Many hypotheses have the same hypernyms such as the leader in Table 2, so we also report the macro accuracy of direction detection averaged across every hypernym in the hypotheses.

The task is challenging because all the candidates have a word-level entailment relation if their compositional meaning is ignored. Furthermore, we cannot infer the entailment direction based on the tendency that longer sentence patterns tend to be more specific because most of the candidate pairs in this dataset have the same length.

As described in Section 2.5, our models detect the direction by computing Ours Diff as

\begin{tabular}{|c|c|cc|}
\hline \multirow{2}{*}{ Method } & Classification & \multicolumn{2}{|c|}{ Direction Detection } \\
\cline { 2 - 4 } & AP@ all & Micro Acc & Macro Acc \\
\hline Random & 21.9 & 50.0 & 50.0 \\
Freq Diff & 21.4 & 54.5 & 47.3 \\
CUSchema & 31.2 & 50.0 & 50.0 \\
Ours (Single) & 23.6 & 50.0 & 50.0 \\
Ours & $\mathbf{3 7 . 8}$ & $\mathbf{6 3 . 1}$ & $\mathbf{5 5 . 4}$ \\
\hline
\end{tabular}

Table 3: Comparison of entailment detection methods. AP and Acc are average precision and accuracy, respectively. All numbers are \%. Our methods use a Transformer as their encoder.

$\operatorname{Asym}\left(\left\{\underline{\tilde{s}}_{i, k}\right\},\left\{\underline{\tilde{s}}_{j, m}\right\}\right)-\operatorname{Asym}\left(\left\{\underline{\tilde{s}}_{j, m}\right\},\left\{\underline{\tilde{s}}_{i, k}\right\}\right)$ and predict the $i$ th sentence pattern to be premise if Ours Diff $>0$. When performing entailment classification, we use as the asymmetric similarity scores $\operatorname{Asym}\left(\left\{\underline{\tilde{s}}_{i, k}\right\},\left\{\underline{\tilde{s}}_{j, m}\right\}\right)$. We report the performance of Ours (Trans), which is the same best model in the RE experiment.

In entailment classification, we compare the results with cosine similarity from Ours (SingleTrans) and CUschema. We also test the frequency difference, which is a strong baseline in hypernym direction detection (Chang et al., 2018). Freq Diff $=\operatorname{Freq}\left(S_{j}\right)-\operatorname{Freq}\left(S_{i}\right)$ where Freq $\left(S_{i}\right)$ is the number of unique entity pairs co-occurred with the $i$ th sentence pattern. The baseline predicts $S_{i}$ to be premise if Freq Diff $>0$ because more general sentence patterns should co-occur with more entity pairs. As a reference, we also report the performance of random scores.

Results: The quantitative and qualitative comparison are presented in Table 3 and Table 2, respectively. Our model that uses multi-facet embeddings significantly outperforms the other baselines. We hypothesize that a major reason is that the sentence patterns with an entailment relation are often similar in some but not all of the facets, and our asymmetric similarity measurement is better at capturing the facet overlapping. 


\section{Related Work}

Relation extraction (RE) is widely studied. Han et al. (2020) summarize the trend of recent studies and point out one of the major challenges is the cost of collecting the labels. Distant supervision (Mintz et al., 2009) and its follow-up work enable us to collect a large amount of training data at a low cost, but the violation of its assumptions often introduces substantial noise into the supervision signal. Our goal is to alleviate the noise issue by representing every sentence pattern using multiple embeddings.

Other noise reduction methods have also been proposed (Roth et al., 2013). For instance, we can adopt multi-instance learning techniques (Yao et al., 2010; Surdeanu et al., 2012; Amin et al., 2020), global topic model (Alfonseca et al., 2012), or both (Roth and Klakow, 2013). We can also reduce the noise by counting the number of shared entity pairs between a sentence pattern and a KB relation (Takamatsu et al., 2012; Su et al., 2018). Nevertheless, the studies focus on mitigating the noise caused by assuming similarity between the sentence patterns and KB relations that co-occur with the same entity pairs, while our method can also reduce the noise from two sentence patterns sharing the same entity pair. Besides, our method is complementary to popular noise reduction methods because our improvement is shown in the training data that have been cleaned (Verga et al., 2016).

Our method is conceptually related to some studies for lexical semantics. For example, word sense induction or unsupervised hypernymy detection can be addressed by clustering the co-occurring words (Neelakantan et al., 2014; Athiwaratkun and Wilson, 2017; Chang et al., 2018). However, the clustering-based methods do not apply to RE because the co-occurring matrix for RE is much sparser (see Section 2.4 for more details).

Finally, our work is inspired by Chang et al. (2021), but they focus on improving the sentence representation rather than RE. We encourage the facet embeddings to become the centers in Kmeans clustering instead of NNSC (non-negative sparse coding) clustering used in Chang et al. (2021), due to its simplicity, efficiency, and better RE performance. Moreover, we discover that an additional regularization described in the appendixis crucial to overcome the sparsity challenge in RE.

\section{Conclusion}

In this work, we address the limitation of representing each sentence pattern using only a single embedding, and our approach improves the distantlysupervised RE performances of compositional universal schema.

Relying on only a very sparse co-occurrence matrix between the sentence patterns and entity pairs, we show that it is possible to predict reasonable cluster centers of entity pair embeddings and to predict the entailment relation between two sentence patterns without any labels.

\section{Acknowledgements}

We thank Ge Gao for the preliminary exploration of this project. We also thank the anonymous reviewers for their constructive feedback.

This work was supported in part by the Center for Data Science and the Center for Intelligent Information Retrieval, in part by the Chan Zuckerberg Initiative under the project Scientific Knowledge Base Construction, in part using high performance computing equipment obtained under a grant from the Collaborative R\&D Fund managed by the Massachusetts Technology Collaborative, in part by the National Science Foundation (NSF) grant numbers DMR-1534431 and IIS-1514053.

Any opinions, findings, conclusions, or recommendations expressed in this material are those of the authors and do not necessarily reflect those of the sponsor.

\section{References}

Kosuke Akimoto, Takuya Hiraoka, Kunihiko Sadamasa, and Mathias Niepert. 2019. Crosssentence n-ary relation extraction using lower-arity universal schemas. In Proceedings of the 2019 Conference on Empirical Methods in Natural Language Processing and the 9th International Joint Conference on Natural Language Processing (EMNLP-IJCNLP), pages 6225-6231, Hong Kong, China. Association for Computational Linguistics.

Enrique Alfonseca, Katja Filippova, Jean-Yves Delort, and Guillermo Garrido. 2012. Pattern learning for relation extraction with a hierarchical topic model. In Proceedings of the 50th Annual Meeting of the Association for Computational Linguistics (Volume 2: Short Papers), pages 54-59, Jeju Island, Korea. Association for Computational Linguistics.

Saadullah Amin, Katherine Ann Dunfield, Anna Vechkaeva, and Guenter Neumann. 2020. A datadriven approach for noise reduction in distantly su- 
pervised biomedical relation extraction. In Proceedings of the 19th SIGBioMed Workshop on Biomedical Language Processing, pages 187-194, Online. Association for Computational Linguistics.

Ben Athiwaratkun and Andrew Wilson. 2017. Multimodal word distributions. In Proceedings of the 55th Annual Meeting of the Association for Computational Linguistics (Volume 1: Long Papers), pages 1645-1656, Vancouver, Canada. Association for Computational Linguistics.

Livio Baldini Soares, Nicholas FitzGerald, Jeffrey Ling, and Tom Kwiatkowski. 2019. Matching the blanks: Distributional similarity for relation learning. In Proceedings of the 57th Annual Meeting of the Association for Computational Linguistics, pages 2895-2905, Florence, Italy. Association for Computational Linguistics.

Kurt D. Bollacker, Colin Evans, Praveen Paritosh, Tim Sturge, and Jamie Taylor. 2008. Freebase: a collaboratively created graph database for structuring human knowledge. In Proceedings of the ACM SIG$M O D$ International Conference on Management of Data, SIGMOD 2008, Vancouver, BC, Canada, June 10-12, 2008, pages 1247-1250. ACM.

Ingwer Borg and Patrick JF Groenen. 2005. Modern multidimensional scaling: Theory and applications. Springer Science \& Business Media.

Arun Chaganty, Ashwin Paranjape, Percy Liang, and Christopher D. Manning. 2017. Importance sampling for unbiased on-demand evaluation of knowledge base population. In Proceedings of the 2017 Conference on Empirical Methods in Natural Language Processing, pages 1038-1048, Copenhagen, Denmark. Association for Computational Linguistics.

Haw-Shiuan Chang, Amol Agrawal, and Andrew McCallum. 2021. Extending multi-sense word embedding to phrases and sentences for unsupervised semantic applications. In Proceedings of the TwentySeventh AAAI Conference on Artificial Intelligence.

Haw-Shiuan Chang, Abdurrahman Munir, Ao Liu, Johnny Tian-Zheng Wei, Aaron Traylor, Ajay Nagesh, Nicholas Monath, Patrick Verga, Emma Strubell, and Andrew McCallum. 2016. Extracting multilingual relations under limited resources: TAC 2016 cold-start KB construction and slot-filling using compositional universal schema. In Proceedings of the 2016 Text Analysis Conference, TAC 2016, Gaithersburg, Maryland, USA, November 1415,2016 . NIST.

Haw-Shiuan Chang, Ziyun Wang, Luke Vilnis, and Andrew McCallum. 2018. Distributional inclusion vector embedding for unsupervised hypernymy detection. In Proceedings of the 2018 Conference of the North American Chapter of the Association for Computational Linguistics: Human Language Technologies, Volume 1 (Long Papers), pages 485-495, New
Orleans, Louisiana. Association for Computational Linguistics.

Rajarshi Das, Manzil Zaheer, Siva Reddy, and Andrew McCallum. 2017. Question answering on knowledge bases and text using universal schema and memory networks. In Proceedings of the 55th Annual Meeting of the Association for Computational Linguistics (Volume 2: Short Papers), pages 358365, Vancouver, Canada. Association for Computational Linguistics.

Xu Han, Tianyu Gao, Yankai Lin, Hao Peng, Yaoliang Yang, Chaojun Xiao, Zhiyuan Liu, Peng Li, Jie Zhou, and Maosong Sun. 2020. More data, more relations, more context and more openness: A review and outlook for relation extraction. In Proceedings of the 1st Conference of the Asia-Pacific Chapter of the Association for Computational Linguistics and the 10th International Joint Conference on Natural Language Processing, pages 745-758, Suzhou, China. Association for Computational Linguistics.

Trevor Hastie, Robert Tibshirani, and Jerome H. Friedman. 2009. The Elements of Statistical Learning. Data Mining, Inference, and Prediction, 2nd Edition. Springer Series in Statistics. Springer.

Sepp Hochreiter and Jürgen Schmidhuber. 1997. Long short-term memory. Neural Comput., 9(8):17351780 .

George A Miller. 1998. WordNet: An electronic lexical database. MIT press.

Mike Mintz, Steven Bills, Rion Snow, and Daniel Jurafsky. 2009. Distant supervision for relation extraction without labeled data. In Proceedings of the Joint Conference of the 47th Annual Meeting of the ACL and the 4th International Joint Conference on Natural Language Processing of the AFNLP, pages 1003-1011, Suntec, Singapore. Association for Computational Linguistics.

Arvind Neelakantan, Jeevan Shankar, Alexandre Passos, and Andrew McCallum. 2014. Efficient nonparametric estimation of multiple embeddings per word in vector space. In Proceedings of the 2014 Conference on Empirical Methods in Natural Language Processing (EMNLP), pages 1059-1069, Doha, Qatar. Association for Computational Linguistics.

Bethany Percha and Russ B. Altman. 2018. A global network of biomedical relationships derived from text. Bioinform., 34(15):2614-2624.

Sebastian Riedel, Limin Yao, Andrew McCallum, and Benjamin M. Marlin. 2013. Relation extraction with matrix factorization and universal schemas. In Proceedings of the 2013 Conference of the North American Chapter of the Association for Computational Linguistics: Human Language Technologies, pages 74-84, Atlanta, Georgia. Association for Computational Linguistics. 
Benjamin Roth, Tassilo Barth, Michael Wiegand, and Dietrich Klakow. 2013. A survey of noise reduction methods for distant supervision. In Proceedings of the 2013 workshop on Automated knowledge base construction.

Benjamin Roth and Dietrich Klakow. 2013. Featurebased models for improving the quality of noisy training data for relation extraction. In 22nd ACM International Conference on Information and Knowledge Management, CIKM'13, San Francisco, CA, USA, October 27 - November 1, 2013, pages 1181-1184. ACM.

Vered Shwartz, Enrico Santus, and Dominik Schlechtweg. 2017. Hypernyms under siege: Linguistically-motivated artillery for hypernymy detection. In Proceedings of the 15th Conference of the European Chapter of the Association for Computational Linguistics: Volume 1, Long Papers, pages 65-75, Valencia, Spain. Association for Computational Linguistics.

Yu Su, Honglei Liu, Semih Yavuz, Izzeddin Gür, Huan Sun, and Xifeng Yan. 2018. Global relation embedding for relation extraction. In Proceedings of the 2018 Conference of the North American Chapter of the Association for Computational Linguistics: Human Language Technologies, Volume 1 (Long Papers), pages 820-830, New Orleans, Louisiana. Association for Computational Linguistics.

Mihai Surdeanu, Julie Tibshirani, Ramesh Nallapati, and Christopher D. Manning. 2012. Multi-instance multi-label learning for relation extraction. In Proceedings of the 2012 Joint Conference on Empirical Methods in Natural Language Processing and Computational Natural Language Learning, pages 455465, Jeju Island, Korea. Association for Computational Linguistics.

Shingo Takamatsu, Issei Sato, and Hiroshi Nakagawa. 2012. Reducing wrong labels in distant supervision for relation extraction. In Proceedings of the 50th Annual Meeting of the Association for Computational Linguistics (Volume 1: Long Papers), pages 721-729, Jeju Island, Korea. Association for Computational Linguistics.

Kristina Toutanova, Danqi Chen, Patrick Pantel, Hoifung Poon, Pallavi Choudhury, and Michael Gamon. 2015. Representing text for joint embedding of text and knowledge bases. In Proceedings of the 2015 Conference on Empirical Methods in Natural Language Processing, pages 1499-1509, Lisbon, Portugal. Association for Computational Linguistics.

Ashish Vaswani, Noam Shazeer, Niki Parmar, Jakob Uszkoreit, Llion Jones, Aidan N. Gomez, Lukasz Kaiser, and Illia Polosukhin. 2017. Attention is all you need. In Advances in Neural Information Processing Systems 30: Annual Conference on Neural Information Processing Systems 2017, December 4 9, 2017, Long Beach, CA, USA, pages 5998-6008.
Patrick Verga, David Belanger, Emma Strubell, Benjamin Roth, and Andrew McCallum. 2016. Multilingual relation extraction using compositional universal schema. In Proceedings of the 2016 Conference of the North American Chapter of the Association for Computational Linguistics: Human Language Technologies, pages 886-896, San Diego, California. Association for Computational Linguistics.

Patrick Verga, Arvind Neelakantan, and Andrew McCallum. 2017. Generalizing to unseen entities and entity pairs with row-less universal schema. In Proceedings of the 15th Conference of the European Chapter of the Association for Computational Linguistics: Volume 1, Long Papers, pages 613-622, Valencia, Spain. Association for Computational Linguistics.

Patrick Verga, Emma Strubell, and Andrew McCallum. 2018. Simultaneously self-attending to all mentions for full-abstract biological relation extraction. In Proceedings of the 2018 Conference of the North American Chapter of the Association for Computational Linguistics: Human Language Technologies, Volume 1 (Long Papers), pages 872-884, New Orleans, Louisiana. Association for Computational Linguistics.

Limin Yao, Sebastian Riedel, and Andrew McCallum. 2010. Collective cross-document relation extraction without labelled data. In Proceedings of the 2010 Conference on Empirical Methods in Natural Language Processing, pages 1013-1023, Cambridge, MA. Association for Computational Linguistics.

Dongxu Zhang, Subhabrata Mukherjee, Colin Lockard, Luna Dong, and Andrew McCallum. 2019. OpenKI: Integrating Open Information Extraction and Knowledge Bases with Relation Inference. In Proceedings of the 2019 Conference of the North American Chapter of the Association for Computational Linguistics: Human Language Technologies, Volume 1 (Long and Short Papers), pages 762-772, Minneapolis, Minnesota. Association for Computational Linguistics. 